\title{
B. Pasternak, Quando rasserena, trad. e cura di A. Niero, Passigli, Bagno a Ripoli 2020, pp. 193.
}

I. L'EDizione. Da un po' di tempo l'editoria italiana ha preso la buona abitudine di pubblicare le raccolte dei grandi poeti russi secondo la conformazione della loro prima apparizione, superando così la fase delle 'scelte', delle 'antologie'. L'editore Passigli nella collana di "Poesia" fondata da Mario Luzi, che ancora nel 2015 aveva pubblicato un florilegio delle poesie d'amore di Boris Pasternak (Anch'io ho conosciuto l'amore. Poesie 1913-1936, a cura di Marilena Rea), viene adesso pubblicando il retaggio poetico del grande scrittore secondo le raccolte col quale era primamente apparso. Così, dopo Temi e variazioni (a cura di Paola Ferretti, 2018), Sui treni del mattino (a cura di Elisa Baglioni, 2019), Mia sorella la vita (a cura di Paola Ferretti, 2020), pubblica adesso l'ultima raccolta poetica, Quando rasserena (a cura di Alessandro Niero, 2020). Nel caso specifico, la pubblicazione riserva al lettore - oltre a poter leggere la raccolta nella sua logica originaria - l'opportunità di affrontare in traduzione italiana l'insieme di questa raccolta, che in precedenza era apparsa solo per frammenti in varie raccolte antologiche di diversi traduttori e, come specificato in nota (p. 21), mai nella sua interezza.

2. IL TESTO. Le liriche della raccolta Kogda razguljaetsja (titolo tradotto anche come Quando torna il sereno, o Quando il tempo si rasserena, e che si potrebbe rendere anche Quando mette al bello [p. I9]) sono state composte tra il I956 e il I959, cioè dopo la stesura del Dottor Živago, comprese le liriche ivi attribuite al protagonista. Questo pone subito al lettore una grossa questione, anzi due: come va visto il ritorno alla composizione in versi dopo la lunga e complessa prova ("l'unica di cui non mi vergogno") del romanzo, e dunque - di nuovo - se Pasternak è un poeta che ha scritto un romanzo, o un prosatore che s'è fatto le ossa scrivendo versi; e se il ritorno alla scrittura di versi sia guidata dalla tensione verso la "semplicità inaudita" del poema Le onde (del 1931), come sembra propendere nella prefazione (p. I3) il curatore. Il quale nella stessa pagina motiva con l'impressione che qui il poeta si sia "scollato dallo sgargiante groviglio metaforico-metonimico delle sue prime raccolte", il che riconduce a una vecchia ma sempre intrigante questione posta da Roman Jakobson, il quale scorgeva nelle prime prove, con l'avallo dello stesso Autore (in una pagina de La reazione di Wassermann, 1914) la predilezione per la metonimia sulla metafora. Al lettore l'ardua sentenza (ma anche qui fanno capolino costruzioni sostanzialmente metonimiche), che comporta un non ovvio esercizio esegetico. Un altro interrogativo, si porrà il lettore italiano, meglio se in grado di leggere il testo a fronte: qual è il valore di queste liriche? Qui mi limito a dire la mia, cioè che più delle poesie "civili" (Vo vsem mne chočetsja..., Byt'znamenitym..., Nobel'skaja premija) gratificate dalla grande no- 
torietà, a me sembra che il meglio sia espresso di nuovo dal senso del vivere còlto attraverso il vivere della natura, mettendo in prima fila Vento (Quattro frammenti su Blok), del 1956.

3. LA TRADUZIONE. Come dichiara con franchezza il traduttore (p.19), egli ha rinunciato alla riproduzione degli schemi rimari, scegliendo di avvalersi per lo più d'una struttura metrica, peraltro non sempre corrispondente nella sua conformazione a quella del russo. Il risultato è generalmente gradevole, anche se talora non si capisce bene l'impiego di un lessico colloquiale, discorsivo (“... al nocciolo, al nucleo", p. 27) per rendere una dizione filosofico-astratta ("do samoj suti”, all'essenza stessa), e talaltra l'alternarsi di metri nella stessa lirica pare accostare la traduzione più a una prosa ritmica che alla resa dell'impianto tonico-sillabica dell'originale (Arano, p. 153). In ogni caso, si tratta di scelte linguistiche consapevoli e meditate (che riferirei al punto c., "decisione non necessaria e motivata”, della tassonomia elaborata da J. Levý) atte, nell' insieme, a offrire al lettore italiano un' immagine convincente di questo tardo Pasternak-poeta.

Cesare G. De Michelis 